\title{
COMPLETE CONVERGENCES AND STRONG LAWS OF LARGE NUMBERS FOR WEIGHTED SUMS PAIRWISE NQD RANDOM VARIABLES SEQUENCE
}

\author{
GuAng-Hui CAI
}

\begin{abstract}
Let $\left\{X_{n}, n \geq 1\right\}$ be a sequence of pairwise NQD random variables. Some complete convergences and strong laws of large numbers for a weighted sums sequence of pairwise NQD random variables are obtained. The results obtainted generalize the results of Cabrera and Volodin (see [3]).
\end{abstract}

\section{INTRODUCTION}

Let $\left\{X, X_{n}, n \geq 1\right\}$ be a sequence of independent identically distributed (i.i.d) random variables. The Marcinkiewicz-Zygmund strong laws of large numbers (SLLN) provides that

$$
\frac{1}{n^{1 / \alpha}} \sum_{i=1}^{n}\left(X_{i}-E X_{i}\right) \rightarrow 0 \text { a.s.for } 1 \leq \alpha<2
$$

and

$$
\frac{1}{n^{1 / \alpha}} \sum_{i=1}^{n} X_{i} \rightarrow \text { 0a.s.for } 0<\alpha<1
$$

if and only if $E|X|^{\alpha}<\infty$. The case $\alpha=1$ is due to Kolmogorov.

As for negatively associated (NA) random variables, Joag and Proschan [8] gave the following definition.

Received 21 June 2005, revised 7 August 2006, accepted 8 August 2006 2000 Mathematics Subject Classification: 60F 15.

Key words and Phrases: NQD, NA, Strong laws of large numbers, Complete convergences, Weighted sums. 
Definition 1.1. [8] A finite family of random variables $\left\{X_{i}, 1 \leq i \leq n\right\}$ is said to be negatively associated (NA) if for every pair of disjoint subsets $T_{1}$ and $T_{2}$ of $\{1,2, \ldots, n\}$, we have

$$
\operatorname{Cov}\left(f_{1}\left(X_{i}, i \in T_{1}\right), f_{2}\left(X_{j}, j \in T_{2}\right)\right) \leq 0,
$$

whenever $f_{1}$ and $f_{2}$ are coordinatewise increasing and the covariance exists. An infinite family is negatively associated if every finite subfamily is negatively associated.

Let $\left\{X, X_{n}, n \geq 1\right\}$ be an identically distributed NA sequence, Matula [10] proved the Kolmogorov strong laws of large numbers. Two random variables $X$ and $Y$ are negative quadrant dependent (NQD), if for all $x, y \in R$, we have

$$
P(X>x, Y>y) \leq P(X>x) P(Y>y) .
$$

A random variables sequence $\left\{X_{k}, k \in N\right\}$ is said to be pairwise NQD, if for all $i \neq j, X_{i}$ and $X_{j}$ are NQD.

The concept of NQD was given by Lehmann [9]. We known that NQD is more general than NA. So NQD is very general. As for pairwise NQD random variables sequences, Wu [13] Proved Kolmogorov strong law of large numbers and complete convergence for pairwise NQD random sequences. Cabrera and Volodin [3] obtained mean convergence theorems and weak laws of large numbers for weighted sums of random variables under a condition of weighted integrability. The result of Cabrera and Volodin [3] sees the following Theorem.

Theorem A Let $\left\{X_{n k}, u_{n} \leq k \leq v_{n}, n \geq 1\right\}$ be an array of rowwise pairwise $N Q D$ random variables and $\left\{a_{n k}, u_{n} \leq k \leq v_{n}, n \geq 1\right\}$ be an array of constants with $\sum_{k=u_{n}}^{v_{n}}\left|a_{n k}\right| \leq C$ for all $n \in \bar{N}$ and some constant $C>0$. Let moreover $\{h(n), n \geq 1\}$ be an increasing sequence of positive constants with $h_{n} \uparrow \infty$ as $n \uparrow \infty$. Suppose that

(a) $\left\{X_{n k}, u_{n} \leq k \leq v_{n}, n \geq 1\right\}$ is $h$ - integrable concerning the array of constants $\left\{a_{n k}\right\}$,

(b) $h^{2}(n) \sum_{k=u_{n}}^{v_{n}} a_{n k}^{2} \rightarrow 0$ as $n \rightarrow \infty$.

Let $T_{n}=\sum_{k=u_{n}}^{v_{n}} a_{n k} X_{n k}$, for all $n \geq 1$. Then $\lim _{n \rightarrow \infty}\left|T_{n}\right|=0$ in Probability.

The main purpose of this paper is to establish some complete convergences and strong laws of large numbers for a weighted sums sequence of pairwise NQD random variables. The results obtainted generalize the results of Cabrera and Volodin [3]. 


\section{MAIN RESULTS}

Throughout this paper, $C$ will represent a positive constant though its value may change from one appearance to the next, and $a_{n}=O\left(b_{n}\right)$ will mean $a_{n} \leq C b_{n}$. In order to prove our results, we need the concept of the Hsu-Robbins-Erdös law of large numbers (see [6], [7]) and the following lemma. Let $\left\{X, X_{n}, n \geq 1\right\}$ be a sequence of independent identically distributed (i.i.d) random variables and denote $S_{n}=\sum_{i=1}^{n} X_{i}$. The Hsu-Robbins-Erdös law of large numbers (see [6], [7]) states that

$$
\forall \varepsilon>0, \sum_{n=1}^{\infty} P\left(\left|S_{n}\right|>\varepsilon n\right)<\infty
$$

is equivalent to $E X=0, E X^{2}<\infty$.

This is a foundamental theorm in probability theory and has been intensively investigated by many authors in the past decades. One of the most important results is Baum and Katz [1] law of large numbers, which states that, for $p<2$ and $r \geq p$

$$
\forall \varepsilon>0, \sum_{n=1}^{\infty} n^{\frac{r}{p}-2} P\left(\left|S_{n}\right|>\varepsilon n^{\frac{1}{p}}\right)<\infty
$$

if and only if $E|X|^{r}<\infty, r \geq 1$, and $E X=0$. There have been many extensions in various directions. Two of them are Chow and Lai ([4], [5]) proposed a two sided estimate and Petrov [11].

Lemma 2.1. [9] Let $X$ and $Y$ are $N Q D$, then

(i) $E X Y \leq E X E Y$;

(ii) $P(X>x, Y>y) \leq P(X>x) P(Y>y)$, for all $x, y \in R$

(iii) if $f(x), g(x)$ are non-decreasing (or non-increasing) functions, then $f(X), g(X)$ are also $N Q D$.

Lemma 2.2. Let $\left\{Y_{i}, i \geq 1\right\}$ be a sequence of centered pairwise $N Q D$ random variables and $E\left|Y_{i}\right|^{2}<\infty$ for every $i \geq 1$. Then there exists $C$, such that

$$
\begin{gathered}
E\left|\sum_{i=1}^{n} Y_{i}\right|^{2} \leq \sum_{i=1}^{n} E\left|Y_{i}\right|^{2} \\
E \max _{1 \leq k \leq n}\left|\sum_{i=1}^{k} Y_{i}\right|^{2} \leq C \log ^{2} n \sum_{i=1}^{n} E\left|Y_{i}\right|^{2}
\end{gathered}
$$

Proof. By Lemma 2.1 and $E Y_{i}=0$, then

$$
E\left|\sum_{i=1}^{n} Y_{i}\right|^{2} \leq \sum_{i=1}^{n} E\left|Y_{i}\right|^{2}+2 \sum_{1 \leq i<j \leq n} E Y_{i} E Y_{j} \leq \sum_{i=1}^{n} E\left|Y_{i}\right|^{2} .
$$


By $E\left|\sum_{i=1}^{n} Y_{i}\right|^{2} \leq \sum_{i=1}^{n} E\left|Y_{i}\right|^{2}$ and Theorem 2.4.1 in Stout [12], we have

$$
E \max _{1 \leq k \leq n}\left|\sum_{i=1}^{k} Y_{i}\right|^{2} \leq C\left(\frac{\log (2 n)}{\log 2}\right)^{2} \sum_{i=1}^{n} E\left|Y_{i}\right|^{2} \leq C \log ^{2} n \sum_{i=1}^{n} E\left|Y_{i}\right|^{2} .
$$

Now we give the concept of $\left\{X_{n k}, u_{n} \leq k \leq v_{n}, n \geq 1\right\}$ is $h$ - integrable concerning the array of constants $\left\{a_{n k}, u_{n} \leq k \leq v_{n}, n \geq 1\right\}$.

Definition 2.3. [3] Let $\left\{X_{n k}, u_{n} \leq k \leq v_{n}, n \geq 1\right\}$ be an array of random variables and $\left\{a_{n k}, u_{n} \leq k \leq v_{n}, n \geq 1\right\}$ an array of constants with $\sum_{k=u_{n}}^{v_{n}}\left|a_{n k}\right| \leq$ $C$ for all $n \in N$ and some constant $C>0$. Let moreover $\{h(n), n \geq 1\}$ be an increasing sequence of positive constants with $h_{n} \uparrow \infty$ as $n \uparrow \infty$. The array $\left\{X_{n k}, u_{n} \leq k \leq v_{n}, n \geq 1\right\}$ is said to be $h$ - integrable concerning the array of constants $\left\{a_{n k}, u_{n} \leq k \leq v_{n}, n \geq 1\right\}$ if the following two conditions hold:

$$
\lim _{n \rightarrow \infty} \sup _{n \geq 1} \sum_{k=u_{n}}^{v_{n}}\left|a_{n k}\right| E\left|X_{n k}\right|<\infty
$$

and

$$
\lim _{n \rightarrow \infty} \sum_{k=u_{n}}^{v_{n}}\left|a_{n k}\right| E\left|X_{n k}\right| I\left(\left|X_{n k}\right|>h(n)\right)=0
$$

We inspired by the concept of $\left\{X_{n k}, u_{n} \leq k \leq v_{n}, n \geq 1\right\}$ is $h$ - integrable concerning the array of constants $\left\{a_{n k}, u_{n} \leq k \leq v_{n}, n \geq 1\right\}$ that we get the following Theorem.

Theorem 2.4 Let $\left\{X_{n k}, u_{n} \leq k \leq v_{n}, n \geq 1\right\}$ be an array of rowwise pairwise $N Q D$ random variables and $\left\{a_{n k}, u_{n} \leq k \leq v_{n}, n \geq 1\right\}$ be an array of constants with $\sum_{k=u_{n}}^{v_{n}}\left|a_{n k}\right| \leq C$ for all $n \in N$ and some constant $C>0$. Let moreover $\{h(n), n \geq 1\}$ be an increasing sequence of positive constants with $h_{n} \uparrow \infty$ as $n \uparrow \infty$. Suppose that

(a) $\left\{X_{n k}, u_{n} \leq k \leq v_{n}, n \geq 1\right\}$ is $h$ - integrable concerning the array of constants $\left\{a_{n k}\right\}$,

(b) $h^{2}(n) \sum_{k=u_{n}}^{v_{n}} a_{n k}^{2} \rightarrow 0$ as $n \rightarrow \infty$,

(c) $h(n) \geq C(\log n)^{1+\delta}$ for some $\delta>0$.

Let $T_{n}=\sum_{k=u_{n}}^{v_{n}} a_{n k} X_{n k}$, for all $n \geq 1$ and $E X_{n k}=0$, for all $n \geq 1, u_{n} \leq k \leq v_{n}$. Then

$$
\forall \varepsilon>0, \sum_{n=1}^{\infty} n^{-1} P\left(\max _{1 \leq j \leq n}\left|T_{j}\right|>\varepsilon\right)<\infty
$$

Proof. $\forall i \geq 1$, define $X_{i}^{(n)}=X_{n i} I\left(\left|a_{n i} X_{n i}\right| \leq h(n)\right)+h(n) I\left(\left|a_{n i} X_{n i}\right|>h(n)\right)-$ $h(n) I\left(\left|a_{n i} X_{i}\right|<-h(n)\right), T_{j}^{(n)}=\sum_{i=1}^{j}\left(X_{i}^{(n)}-E X_{i}^{(n)}\right)$, 
then $\forall \varepsilon>0$,

$$
P\left(\max _{1 \leq j \leq n}\left|T_{j}\right|>\varepsilon\right) \leq P\left(\max _{1 \leq j \leq n}\left|a_{n j} X_{n j}\right|>h(n)\right)+P\left(\max _{1 \leq j \leq n}\left|T_{j}^{(n)}\right|>\varepsilon-\max _{1 \leq j \leq n}\left|\sum_{i=1}^{j} E X_{i}^{(n)}\right|\right) .
$$

First we show that

$$
\max _{1 \leq j \leq n}\left|\sum_{i=1}^{j} E X_{i}^{(n)}\right| \rightarrow 0, \text { asn } \rightarrow \infty .
$$

In fact, by (a) and (b), then

$$
\begin{aligned}
& \max _{1 \leq j \leq n}\left|\sum_{i=1}^{j} E X_{i}^{(n)}\right| \\
& =\max _{1 \leq j \leq n}\left|\sum_{i=1}^{j} E\left[X_{n i} I\left(\left|a_{n i} X_{n i}\right| \leq h(n)\right)+h(n) I\left(\left|a_{n i} X_{n i}\right|>h(n)\right)-h(n) I\left(\left|a_{n i} X_{n i}\right|<-h(n)\right)\right]\right| \\
& \leq \max _{1 \leq j \leq n}\left|\sum_{i=1}^{j} E X_{n i} I\left(\left|X_{n i}\right|>h(n)\right)\right|+h(n) \sum_{j=1}^{n} P\left(\left|X_{n j}\right|>h(n)\right) \rightarrow 0, \text { asn } \rightarrow \infty .
\end{aligned}
$$

From (2.4), hence (2.3) is true. From (2.2) and (2.3), it follows that for $n$ large enough

$$
P\left(\max _{1 \leq j \leq n}\left|T_{j}\right|>\varepsilon\right) \leq P\left(\max _{1 \leq j \leq n}\left|a_{n j} X_{n j}\right|>h(n)\right)+P\left(\max _{1 \leq j \leq n}\left|T_{j}^{(n)}\right|>\frac{\varepsilon}{2}\right) .
$$

Hence we need only to prove that

$$
\begin{gathered}
I=: \sum_{n=1}^{\infty} n^{-1} \sum_{j=1}^{n} P\left(\left|a_{n j} X_{n j}\right|>h(n)\right)<\infty, \\
I I=: \sum_{n=1}^{\infty} n^{-1} P\left(\max _{1 \leq j \leq n}\left|T_{j}^{(n)}\right|>\frac{\varepsilon}{2}\right)<\infty .
\end{gathered}
$$

By Markov inequality, (a), (b) and (c), it follows easily that

$$
\begin{aligned}
I & =\sum_{n=1}^{\infty} n^{-1} \sum_{j=1}^{n} P\left(\left|a_{n j} X_{n j}\right|>h(n)\right) \\
& \leq \sum_{n=1}^{\infty} n^{-1} \sum_{j=u_{n}}^{v_{n}} P\left(\left|a_{n j} X_{n j}\right|>h(n)\right) \\
& \leq \sum_{n=1}^{\infty} n^{-1} \sum_{j=u_{n}}^{v_{n}} \frac{E\left|a_{n j} X_{n j}\right|}{h(n)} \\
& \leq C \sum_{n=1}^{\infty} n^{-1}(\log n)^{-1-\delta}<\infty .
\end{aligned}
$$


By Markov inequality, Lemma 2.2, (a), (b) and (c), then

$$
\begin{aligned}
I I & =\sum_{n=1}^{\infty} n^{-1} P\left(\max _{1 \leq j \leq n}\left|T_{j}^{(n)}\right|>\frac{\varepsilon}{2}\right) \\
& \leq \sum_{n=1}^{\infty} n^{-1}(\varepsilon / 2)^{-2} E \max _{1 \leq j \leq n}\left|T_{j}^{(n)}\right|^{2} \\
& \leq C \sum_{n=1}^{\infty} n^{-1}(\log n)^{2} \sum_{j=1}^{n} E\left|X_{j}^{(n)}\right|^{2} \\
& \leq C \sum_{n=1}^{\infty} n^{-1}(\log n)^{2} \sum_{i=1}^{n} E\left|X_{n i} I\left(\left|a_{n i} X_{n i}\right| \leq h(n)\right)+h(n) I\left(\left|a_{n i} X_{n i}\right|>h(n)\right)\right|^{2} \\
& <\infty .
\end{aligned}
$$

Now we complete the proof of Theorem 2.4.

Corollary 2.5. Under the conditions of Theorem 2.1, then

$$
\lim _{n \rightarrow \infty}\left|T_{n}\right|=0 \quad \text { a.s. }
$$

Proof. By (2.1), we have

$$
\begin{aligned}
\infty & >\sum_{n=1}^{\infty} n^{-1} P\left(\max _{1 \leq j \leq n}\left|T_{j}\right|>\varepsilon\right) \\
& =\sum_{i=0}^{\infty} \sum_{n=2^{i}}^{2^{i+1}-1} n^{-1} P\left(\max _{1 \leq j \leq n}\left|T_{j}\right|>\varepsilon\right) \\
& \geq \frac{1}{2} \sum_{i=1}^{\infty} P\left(\max _{1 \leq j \leq 2^{i}}\left|T_{j}\right|>\varepsilon\right) .
\end{aligned}
$$

By Borel-Cantelli Lemma, we have

$$
P\left(\max _{1 \leq j \leq 2^{i}}\left|T_{j}\right|>\text { ci.o. }\right)=0 .
$$

Hence

$$
\lim _{i \rightarrow \infty} \max _{1 \leq j \leq 2^{i}}\left|T_{j}\right|=0 \quad \text { a.s. }
$$

and using

$$
\max _{2^{i-1} \leq n<2^{i}}\left|T_{n}\right| \leq \max _{1 \leq j \leq 2^{i}}\left|T_{j}\right|,
$$

We have

$$
\lim _{n \rightarrow \infty}\left|T_{n}\right|=0 \quad \text { a.s. }
$$


Now we complete the proof of Corollary 2.5 .

Remark Corollary 2.5. generalizes the results of Cabrera and Volodin [3].

\section{CONCLUDING REMARKS}

In this paper, some complete convergences and strong laws of large numbers for a weighted sums sequence of pairwise NQD random variables are obtained.

Acknowledgement. This research is supported by Key discipline of Zhejiang Province (Key discipline of Statistics of Zhejiang Gongshang University) and National Natural Science Foundation of China.

\section{REFERENCES}

1. L.E. Baum And M. Katz, "Convergence rates in the law of large numbers", Trans. Amer. Math. Soc. 120 (1965), 108-123.

2. A. Bozorgnia, R.F. Patterson and R. L. Taylor, "On the strong laws of large numbers for arrays of rowwise independent random elements", J. Math. and Math. Sci. 16 (1993), 587-592.

3. M.O. Cabrera AND A.I. Volodin, "Mean convergence theorems and weak laws of large numbers for weighted sums of random variables under a condition of weighted integrability", J. Math. Anal. Appl. 305 (2005), 644-658.

4. Y.S. Chow AND T.L. LAI, "Some one-sided theorems on the tail distribution of sample sums with applications to the last time and largest excess of boundary crossings", Trans. Amer. Math. Soc. 208 (1975), 51-72.

5. Y.S. ChOw AND T.L. LAI, "Paley-Type inequalities and convergence rates related to the law of large numbers and extended renewal theory", Z. Wahrsch. Verw. Geb. 45 (1978), 1-19.

6. P. Erdös, "On a theorem of Hsu-Robbins", Ann. Math. Statist. 20 (1949), 286-291.

7. P.L. Hsu And H.Robbins, "Complete convergence and the law of larege numbers", Proc. Nat. Acad. Sci. (USA), 33(2) 1947, 25-31.

8. D.K. JoAg And F. Proschan, "Negative associated of random variables with application", Ann. Math. Statist. 11 (1983), 286-295.

9. E.L. Lehmann, "Some concepts of dependence", Ann. Math. Statist. 37 (1966), $1137-1153$.

10. P. Matula, "A note on the almost sure convergence of sums of negatively dependence random sequences", Statist. Probab. Lett. 15(3) (1992), 209-213.

11. V.V. Petrov, Limit theorems of probability theory sequences of independent random variables, Oxford, Oxford Science Publications, 1995. 
12. W. Stout, Almost sure convergence, New York, Academic Press, 1974.

13. Q.Y. Wu, "Convergence properties of pairwise NQD random sequences", Acta Math. Sinica 45 (2002), 617-624.

Guang-Hui CAI : Department of Mathematics and Statistics, Zhejiang Gongshang University, Hangzhou 310035, P. R. China.

E-mail: cghzju@sohu.com 